\title{
Phase-structural and non-linear effects in heterogeneous systems
}

\author{
D.V. Alexandrov ${ }^{1, a}$ and A.Yu. Zubarev ${ }^{2}$ \\ ${ }^{1}$ Department of Theoretical and Mathematical Physics, Laboratory of Multi-Scale \\ Mathematical Modeling, Ural Federal University, Lenin ave. 51, Ekaterinburg 620000, \\ Russia \\ ${ }^{2}$ Department of Theoretical and Mathematical Physics, Laboratory of Mathematical \\ Modeling of Physical and Chemical Processes in Multiphase Media, Ural Federal \\ University, Lenin ave. 51, Ekaterinburg 620000, Russia
}

Received 23 September 2020 / Accepted 23 September 2020 Published online 19 November 2020

\begin{abstract}
This theme issue is concerned with modern achievements in the theory, computational and experimental studies of the phase transitions that occur in heterogeneous systems. The papers are divided into six thematic sections devoted to patterned formations, transport and magnetic phenomena, hydrodynamic flows as well as biological applications. Special attention is paid to various non-linear effects met in these processes and leading to some unexpected at first glance physical aspects.
\end{abstract}

\section{Introduction}

Structural-phase and nonlinear effects radically affect the dynamic behavior of many heterogeneous systems, determine their internal contexture, composition, morphology, and properties [1-14]. These effects arise as a result of various driving forces or external impacts and require especial theoretical consideration and computational modeling to describe and predict the metastable and non-equilibrium transformations in heterogeneous materials. Here, such phenomena as microstructure formation (e.g. monocrystalline or polycrystalline) during dendritic crystallization with different undercoolings [15-18], crystal-size distribution in metastable liquids induced by external mass and heat sources [19-21], magnetic hyperthermia in tumor cells produced by the alternating external magnetic field [22-24], and circulating fluid flows in coronary vessels appearing behind stenoses may be mentioned $[25,26]$. These and other similar problems attract the attention of many researchers from all over the world. Some of them representing scientific teams from Germany, the United Kingdom, France, Morocco, Egypt, and Russia reported the results of joint scientific research in the present theme issue.

Following the topics of scientific directions, all articles of the theme issue are grouped into six sections, namely,

1. Dendritic growth [27-30];

2. Nucleation and crystal growth [31,32];

a e-mail: dmitri.alexandrov@urfu.ru 
3. Mushy layer [33];

4. Non-equilibrium structuring in magnetic heterogeneous systems [34,35];

5. Theoretical modeling of magnetic hyperthermia [36,37];

6. Transport and non-equilibrium structures in biological systems [38-40].

The first section represents the recent results of collaboration on dendritic growth of the research teams from the Friedrich Schiller University Jena (Germany), the Ural Federal University (Russia), the University of Hassan II Casablanca (Morocco), the University of Greenwich (United Kingdom), and the University of Rouen Normandy (France). Here, the non-linear effect of a tiny amount of impurity on the stable mode of dendritic evolution in binary melts is presented in reference [27], and the selection constants describing such growth are found in reference [28]. More complex nonaxisymmetric dendritic growth with arbitrary crystalline symmetry in two and three dimensions theoretically and numerically studied in reference [29]. In this paper, the sharp interface model is tested against the phase-field simulations. The phase-field model is developed by the effective mobility approach in reference [30] to describe the slow and rapid solidification scenario. In this regard, the phase-field equations are reduced to a hodograph equation for the solid-liquid interface movement, which is applied to the problem of solute trapping in a binary system. Since large undercooling, in addition to dendritic growth, often leads to nucleation and growth of crystallites, the second section is devoted to non-linear effects arising in the study of bulk phase transitions. Here, the answer to the question of how the shift in the phase transition temperature (arising due to the Gibbs-Thomson and attachment kinetics effects) influences the evolution of crystals in metastable liquids is formulated in reference [31]. The effect of the shape of growing crystals, which are often can be approximated by ellipsoidal particles, on the desupercooling kinetics in metastable melts is studied in reference [32]. The third section is a continuation of studies on the evolution of a new phase in a metastable melt, where dendritic growth and nucleation of crystallites can occur simultaneously in the two-phase (mushy) layer. With this aim in view, the theory of directional crystallization with a mushy layer in the presence of vigorous convection is developed in reference [33].

The fourth section presents the results of the theoretical study of non-equilibrium, non-ergodic structuring in magnetic fluids, consisting of nano-and micron-sized magnetic particles, suspended in a carrier liquid. The paper [34] deals with the study of magnetic nano-particles rotation under the action of a running oscillating magnetic field. This problem attracts significant interests because of the perspective of this effect application in bio and medical technologies. In part for therapy of the strokes, thromboses, and other blood vessel diseases. The key idea of this approach is in induction, by the rotating particles and their aggregates, the meso- and macroscopic circulating flow of the host liquid (blood). These circulations intensify the drug delivery to the clot in the vessel that provides the therapeutic effect. The rate and the spatial scale of these circulations are estimated in [34] as a function of the strength and period of the running field, as well as other parameters of the system. The results are obtained in collaboration between the teams of the Ural Federal University and the University of Cote d'Azur (France). The second work [35] of this section presents the results of a collaboration between the Ural Federal University and Technical University of Dresden (Germany). In this paper, aggregation of magnetizable micron-sized particles under the action of the applied stationary magnetic field is studied by using computer simulation. The results demonstrate that under the field action the particles do not form regular column-like structures, as it is supposed in traditional theoretical models, however, unite into stable, but thermodynamically non-equilibrium (nonergodic) branched and net-like structures, percolating the sample from one boundary to the opposite one. Note that suspensions of the magnetizable particles (magnetorheological suspensions) find active applications in modern and perspective technologies as dampers, shock-absorbers, actuators, artificial muscles, etc. due to the ability to 
change, up to several orders of magnitude, their rheological properties with the help of the applied magnetic field. The field effect on the elastic properties of the suspensions with the percolating structures is estimated in the work [35].

Magnetic hyperthermia (MH) is a progressive method of cancer and other tumor diseases. The key idea of this method is in the injection of the biologically intact magnetic nanoparticles into the tumor region and heating of the particles, therefore, the tumor cells, with the help of an alternating magnetic field. If the temperature of the ill cell exceeds some threshold temperature (usually estimated as $42^{\circ} \mathrm{C}$ ), the cell dies. At the same time, the healthy cells are more temperature resistible and survive up to the temperature of $52-55^{\circ} \mathrm{C}$. The $\mathrm{MH}$ method is especially effective in combination with the traditional methods of chemical and radiotherapy. The practical application of $\mathrm{MH}$ therapy requires accurate prediction of the intensity of the heat production by the particles. The works $[36,37]$ are devoted to mathematical modeling and estimation of the effects in the systems of magnetically interacting particles at their various spatial dispositions, which take place at the particles' injection into the cells. These works are done as a result of a collaboration between the Ural Federal University and the Menoufia University (Egypt).

The sixth section is concerned with the formation of heterogeneous structures in complex fluids. The first paper here [38] represents a collaboration between the Ural State Medical University, Tyumen Cardiology Research Center, and the Ural Federal University (located in Russia), where experimental, theoretical, and computational studies, have been carried out. This paper reports results on complex modeling of hemodynamic processes in an artery with stenosis. The paper is devoted to the test simulations for a native artery model on the basis of medical observations. The next article [39], continuing studies of complex flows, considers the combined process of transport and dissolution of microcrystals in a semi-infinite channel (blood vessels). For this purpose, the flux of crystals at a certain channel cross-section, the concentration distribution of crystals, and their dissolution intensity are determined theoretically in the case of well-developed turbulent flow. These studies have great potential for medical applications because the blood flows are important in describing the hydrodynamic processes in living organisms, and they are associated with the pathogenesis of different heart diseases. So, for example, such flows build up the fluid-dynamic shear stress on blood components and encourage platelet aggregation, which is responsible for thrombus evolution in perturbed flow zones. The concluding paper [40] is concerned with the formation of heterogeneous structures due to the diffusion in a non-linear glycolytic model. Here, the authors demonstrate how the temporal oscillations of homogeneous structures transform into the stationary non-homogeneous patterns-attractors under increasing diffusion.

We hope that this issue will be of interest to a wide range of specialists in materials physics. Namely for theoretical physicists and chemists working in an area of phase and structural transformations, for developers of computational algorithms and software programs, for scientists engaged in the field of computational experiments, and, of course, for experimentalists from various areas of heterogeneous materials. The issue proves to be a convenient forum to discuss modern trends and achievements, find new solutions, and strengthen the research collaborations.

In conclusion, we would like to thank all author teams for their remarkable contributions to the present theme issue. We are also grateful to all reviewers for their thorough comments, enabling us to improve the presentation of research works. Finally, we would like to thank the EPJ ST publishing team for useful collaborations in organizing the present theme issue.

This work was supported by the Russian Science Foundation (grant number 18-19-00008).

Publisher's Note The EPJ Publishers remain neutral with regard to jurisdictional claims in published maps and institutional affiliations. 


\section{References}

1. J.W. Mullin, Crystallization (Butterworths, London, 1972)

2. K.F. Kelton, A.L. Greer, Nucleation in condensed matter: applications in materials and biology (Elsevier, Amsterdam, 2008)

3. D.V. Alexandrov, Int. J. Heat Mass Trans. 47, 1383 (2004)

4. K. Libbrecht, Snowflakes (Voyageur Press, Minneapolis, MN, 2004)

5. D.V. Alexandrov, Phil. Mag. Lett. 94, 786 (2014)

6. D.V. Alexandrov, P.K. Galenko, Acta Mater. 137, 64 (2017)

7. V.Ya. Shur, A.R. Akhmatkhanov, Philos. Trans. R. Soc. A 376, 20170204 (2018)

8. P.K. Galenko, D.V. Alexandrov, Philos. Trans. R. Soc. A 376, 20170210 (2018)

9. D.V. Alexandrov, P.K. Galenko, Philos. Trans. R. Soc. A 378, 20190243 (2020)

10. D.V. Alexandrov, A.Yu. Zubarev, Philos. Trans. R. Soc. A 377, 20180353 (2019)

11. D.V. Alexandrov, A.Yu. Zubarev, Philos. Trans. R. Soc. A 378, 20200002 (2020)

12. I.V. Alexandrova, D.V. Alexandrov, Philos. Trans. R. Soc. A 378, 20190245 (2020)

13. O.V. Gusakova, P.K. Galenko, V.G. Shepelevich, D.V. Alexandrov, M. Rettenmayr, Philos. Trans. R. Soc. A 377, 20180204 (2019)

14. P.K. Galenko, D.A. Danilov, K. Reuther, D.V. Alexandrov, M. Rettenmayr, D.M. Herlach, J. Cryst. Growth 457, 349 (2017)

15. D.V. Alexandrov, P.K. Galenko, Physics-Uspekhi 57, 771 (2014)

16. D.V. Alexandrov, P.K. Galenko, L.V. Toropova, Philos. Trans. R. Soc. A 376, 20170215 (2018)

17. P.K. Galenko, D.V. Alexandrov, E.A. Titova, Philos. Trans. R. Soc. A 376, 20170218 (2018)

18. W. Kurz, D.J. Fisher, Fundamentals of solidification (Trans. Tech. Publications, Aedermannsdorf, 1992)

19. D.V. Alexandrov, Chem. Eng. Sci. 117, 156 (2014)

20. E.V. Makoveeva, D.V. Alexandrov, Philos. Trans. R. Soc. A 376, 20170327 (2018)

21. E.V. Makoveeva, D.V. Alexandrov, Philos. Trans. R. Soc. A 377, 20180210 (2019)

22. A.Y. Zubarev, Philos. Trans. R. Soc. A 377, 20180213 (2019)

23. A.F. Abu-Bakr, A.Yu. Zubarev, Philos. Trans. R. Soc. A 378, 20190251 (2020)

24. A.F. Abu-Bakr, A.Yu. Zubarev, Eur. Phys. J. Special Topics 229, 323 (2020)

25. S. Tu, J. Westra, J. Yang et al., JACC: Cardiovasc. Interv. 9, 2024 (2016)

26. W.F. Fearon, S. Achenbach, T. Engstrom et al., Circulation 139, 477 (2019)

27. O.V. Kazak, P.K. Galenko, D.V. Alexandrov, I.O. Starodumov, Eur. Phys. J. Special Topics 229, 2885 (2020)

28. E.A. Titova, D.V. Alexandrov, P.K. Galenko, Eur. Phys. J. Special Topics 229, 2891 (2020)

29. L.V. Toropova, P.K. Galenko, D.V. Alexandrov, M. Rettenmayr, A. Kao, G. Demange, Eur. Phys. J. Special Topics 229, 2899 (2020)

30. A. Salhoumi, D.V. Alexandrov, P.K. Galenko, Eur. Phys. J. Special Topics 229, 2911 (2020)

31. E.V. Makoveeva, D.V. Alexandrov, Eur. Phys. J. Special Topics 229, 2923 (2020)

32. M.A. Nikishina, D.V. Alexandrov, Eur. Phys. J. Special Topics 229, 2937 (2020)

33. D.V. Alexandrov, N.K. Goltyakov, A.A. Ivanov, I.V. Alexandrova, Eur. Phys. J. Special Topics 229, 2951 (2020)

34. A.Yu. Zubarev, M. Raboisson-Michel, G. Verger-Dubois, P. Kuzhir, Eur. Phys. J. Special Topics 229, 2961 (2020)

35. A. Zubarev, D. Chirikov, D. Borin, Eur. Phys. J. Special Topics 229, 2967 (2020)

36. A.F. Abu-Bakr, A.Yu. Zubarev, Eur. Phys. J. Special Topics 229, 2981 (2020)

37. A.F. Abu-Bakr, A.Yu. Zubarev, Eur. Phys. J. Special Topics 229, 2991 (2020)

38. I.O. Starodumov, F.A. Blyakhman, S.Yu. Sokolov, I.S. Bessonov, A.Yu. Zubarev, D.V. Alexandrov, Eur. Phys. J. Special Topics 229, 3009 (2020)

39. D.V. Alexandrov, S.P. Okhezin, A.A. Ivanov, Eur. Phys. J. Special Topics 229, 3021 (2020)

40. I. Bashkirtseva, A. Pankratov, Eur. Phys. J. Special Topics 229, 3033 (2020) 